\title{
Effects of hypothalamic current intensity on object carrying induced by shuttlebox self-stimulation in rats*
}

\author{
JOSEPH MENDELSON and GLEN MAUL $\dagger$ \\ University of Kansas, Lawrence, Kansas 66044
}

\begin{abstract}
Five rats with electrodes in the perifornical lateral hypothalamic area learned to self-stimulate in a shuttlebox by running back and forth to turn the intracranial stimulation (ICS) on and off. When objects (food pellets and wood blocks) were introduced into the box, all the rats developed a tendency to carry (hoard) them from the ON side to the $\mathrm{OFF}$ side. The rate of self-stimulation and the percentage of self-stimulations on which hoarding occurred were studied as a function of ICS intensity (high or low) and the presence or absence of objects. Each rat showed three trends: (1) The rate of self-stimulation decreased at the low intensity, both under the object and the empty condition. (2) This decrement in self-stimulation rate was more pronounced on object sessions than on empty sessions. (3) Percent hoarding decreased at the low intensity. These data are discussed in terms of the "security hypothesis" of hoarding and the aversive properties of long durations of ICS.
\end{abstract}

When rats are required to operate a shuttlebox to obtain intracranial stimulation (ICS) in the lateral hypothalamic area (LHA), some of them develop a tendency to carry objects, such as food pellets, wood blocks, and paper strips, from the ON side of the box to the OFF side (Mendelson, 1966, 1968, 1969, 1972; Phillips et al, 1969). Mendelson (1969, 1972) has interpreted this object carrying in terms of the "security hypothesis," which was previously developed to account for the hoarding behavior manifested by rodents under natural conditions (Bindra, 1948, 1959). According to this hypothesis, hoarding is an expression of a rodent's tendency to transport objects located in insecure and unfamiliar places into the security and safety of the nest or home territory.

A similar territorial analysis can be made of the ON and OFF sides of the shuttlebox. Beer et al (1968) have reported evidence suggesting that rats terminate self-administered trains of hypothalamic ICS because the ICS becomes aversive if permitted to continue for too long durations. If this interpretation of shuttlebox self-stimulation is correct, then it follows that while the rat is learning to turn the ICS on and off, it occasionally receives aversive ICS on the ON side of the shuttlebox. In contrast, it never receives aversive ICS on the extreme end of the OFF side, since the ICS is never on there. This latter part of the box is therefore analogous to the rat's safe home territory, while the rest of the box is similar to outside, unfamiliar territory where aversive events are more probable.

Since the threat of aversive ICS on the ON side of the box renders this side insecure, the rats carry objects from the ON side to the safe OFF side of the box where

*Supported in part by grants to J.M. from the United States Public Health Service (MH 14410 and $\mathrm{MH} 21955$ ) and the National Science Foundation (GB7370).

$\div$ G.M. is now at the Department of Psychology, Brooklyn College, Brooklyn. New York. there is no threat of aversive ICS. According to this analysis, the probability of hoarding is related to the magnitude of the security differential between the ON and OFF sides. Thus, any factor which increases this differential should increase the probability of hoarding.

There is evidence suggesting that an increase in ICS intensity might be such a factor. Beer et al (1968) showed not only that rats would learn to avoid durations of hypothalamic ICS which exceeded those selected by the animals in a self-stimulation situation, but also that the frequency of the avoidance response increased with ICS intensity. This probably means that high-intensity ICS (1) becomes aversive more quickly, and/or (2) becomes more aversive than low-intensity ICS (Stein, 1962). In either case, increasing ICS intensity should increase the security differential between the $\mathrm{ON}$ and OFF sides of the shuttlebox, and thereby increase the probability of hoarding. The present experiment was designed to test this prediction.

\section{METHOD}

\section{Subjects}

The Ss were five male hooded rats, weighing $280-350 \mathrm{~g}$ at the beginning of the experiment. Each rat was stereotaxically implanted while under Equi-Thesin anesthesia with a pair of monopolar stainless steel electrodes aimed at the perifornical area of the lateral hypothalamus. The electrodes were insulated with Stoner-Mudge Packaging Coating (obtained from Mobil Chemical, Pittsburgh. Pennsylvania), except for $0.5 \mathrm{~mm}$ at the tip. A wire leading from four screws mounted in the skull served as indifferent electrode. All these rats had been trained to self-stimulate in the shuttlebox, and their carrying had been observed in the presence of food pellets, wood blocks. paper strips. and rat pups. as previously described (Mendelson. 1972).

\section{Apparatus}

The experiments were conducted in a shuttlebox measuring $80 \times 26 \times 35 \mathrm{~cm}$ high. The bo $\times$ had a Plexiglas floor and three wood walls. and one of the larger walls consisted of a one-way mirror. Photocells and light beams were mounted $15 \mathrm{~cm}$ from 
each end of the box. $4.5 \mathrm{~cm}$ above the floor. When the animal interrupted a beam of light (which travelled across the width of the box to strike one of the photocells). $60-\mathrm{Hz}$ sine-wave ICS was turned on; interruption of the beam of light striking the other photocell terminated the ICS.

\section{Procedure}

The rats were trained to shuttle and to carry objects. as previously described (Mendelson, 1972). Then. for each animal, an intensity was found which resulted in a mean self-selected ON duration of $5-10 \mathrm{sec}$ in the presence of Purina food pellets and wood blocks of the same size.

Each animal was then given two 10 -min sessions on each of 8 days (Rat E4 was tested for 12 days). The sessions were separated by at least $2 \mathrm{~h}$. On alternate days, each rat was tested in the empty box or with objects present. On each day, one session was conducted at the previously determined intensity and the other session was conducted at an intensity which was reduced from this by $14 \%-22 \%$. The order in which these daily sessions were administered was reversed after the 1st. 3rd, and 6 th day's (and also after the 9th and 11 th days for Rat E4), so that for each environmental condition (empty and objects), each intensity was used on the first of the two daily sessions as of ten as it was used on the second of these sessions. The precise order used for each of four of the rats is indicated in Figs. 1-4.

At the conclusion of the experiment, the animals were perfused with $10 \%$ formal-saline, and their brains were removed and sliced at 50 microns. The wet sections were projected directly onto photographic paper, which was then developed. Accurate placements could be determined for only four of the animals: their electrodes were found to lie in the perifornical LHA at the anterior-posterior level of the ventromedial nucleus of the hypothalamus (DeGroot A5.0-A6.2).

\section{RESULTS}

For three rats, the percent of self-stimulations (round-trip crossings) on which hoarding occurred decreased at the lower intensity. For the other two rats, hoarding remained close to $100 \%$ at both intensities. Therefore, their lower intensity was decreased by an additional $2 \mu \mathrm{A}$ (after 1 day of testing for Rat E4 and after 4 days for E20). This new low intensity brought about a decrement in percent hoarding in both of the rats. The sequence of tests was therefore started anew. Rat C15 showed a large decrement in hoarding at the lower intensity, but also developed a decrement on its second session at the high intensity (only $25 \%$ hoarding). Its high intensity was therefore raised by $3 \mu \mathrm{A}$, and the sequence of tests was started anew. Rat D12 developed a large tumor on the back of its head, and its hoarding at the high intensity dropped to $15 \%$ on the last day of testing; raising the intensity did not reinstate hoarding. On all previous pairs of tests with objects, its percent hoarding was higher at the high intensity. Autopsy revealed tumors throughout the thoracic cavity as well.

The results for four of the five rats are shown in Figs. 14. In each figure, three trends can be detected: (1) Percent hoarding decreased at the low intensity (compare the thick black bars, representing percent hoarding at the high intensity, with the thick striped bars, representing hoarding at the low intensity). (2) The number of self-stimulations (crossings) tended to decrease at the low intensity both under the object and the empty conditions (object condition: compare the thin black bars. representing the number of crossings at the high intensity. with the thin striped black bars. representing the number of crossings at the low intensity: empty condition: compare the plain white bars with the striped white bars). (3) The availability of objects in the shuttlebox increased the number of self-stimulations more at the high intensity than at the low intensity (the difference in height between the thin plain black bars and the thin plain white bars is greater than the difference between the thin striped black bars and the thin striped whited bars). Another way of expressing this same trend is to say that the lower intensity decreased self-stimulations more on object sessions than on empty sessions. This relationship is probably a reflection of the fact that the objects induced more hoarding at the high intensity and that hoarding tends to greatly increase the rate of shuttlebox self-stimulation (Mendelson, 1966, 1968, 1969. 1972: Phillips et al, 1969).

For each of the four rats whose data are depicted in the figures, each of the first two trends was evaluated statistically by a two-tailed t test and was found to be significant at the $p<.05$ with two exceptions: for Rats E20 and E24, the decrease in self-stimulations brought about by decreasing ICS intensity was not significant under the empty condition. Thus, when these two rats were tested under the empty condition, the low intensity supported the same rate of self-stimulation as the high intensity. If these rats had only been tested under the empty condition, we would have concluded that the small difference between the two intensities of current had no significant effect on shuttling for ICS. However, under the object condition, we discovered that the low intensity induced large decrements in carrying behavior, while the high intensity supported a high level of carrying behavior, and this, in turn, led to a large increase in self-stimulation rate. In a sense, the introduction of objects into the shuttlebox served to amplify or sensitize the testing situation to the effects of ICS intensity on self-stimulation rate.

This effect constitutes the third trend mentioned above, i.e., the interaction effect. An analysis of variance was performed to ascertain the significance of the interaction between intensity and the presence or absence of objects in the shuttlebox. A mean score for each $S$ was computed for each condition, and the analysis of variance was performed on these scores (Intensity by Object Condition by S). The error term was obtained by pooling all $\mathrm{S}$ interaction terms. For the intensity effect, $F=111.52, \mathrm{df}=1 / 9 \mathrm{p}<.01$. For the object condition, $F=17.67, \mathrm{df}=1 / 9, \mathrm{p}<.01$. The interaction between intensity and object condition yielded an $F=29.08, d f=9, p<.01$. The nature of this interaction was evaluated by Fisher's lsd procedure $(p=$ $.01)$. This test revealed a significant tendency for the availability of objects to increase the number of 
Figs. 1-4. Each rat was self-stimulating in a shuttlebox at either a low (striped bars) or high (plain bars) intensity of hypothalamic ICS: on half of the sessions, at each intensity, food and wood objects $(0)$ were available in the box (black bars). On the other sessions, the box was empty (E, white bars). For each 10 -min object session, the wide bars indicate the percentage of round-trip crossings (self-stimulations) on which an object was carried (hoarded) from the ON side to the OFF side of the box. The thin bars indicate the number of crossings made on each object and empty session.
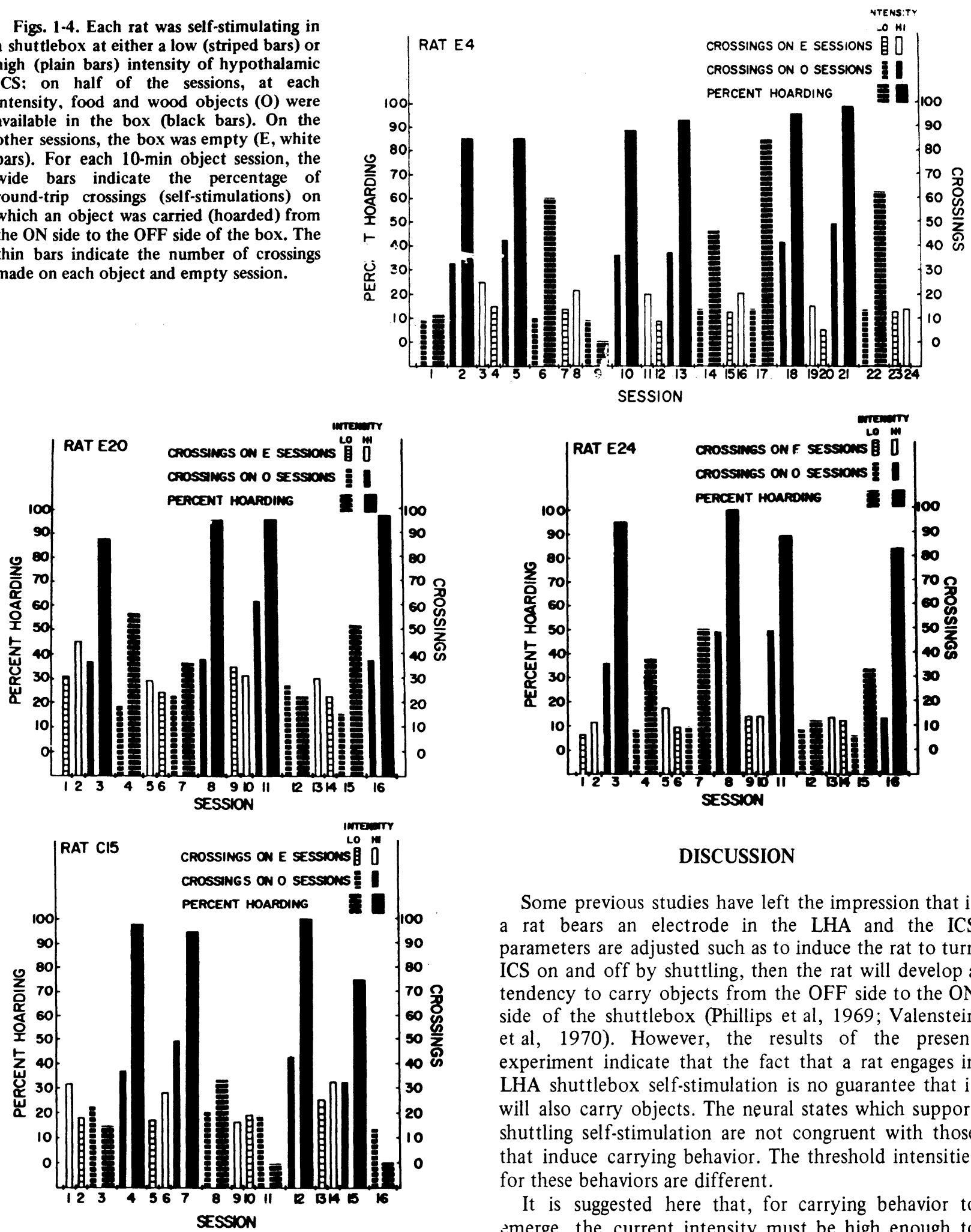

self-stimulations more at the high than at the low intensity.

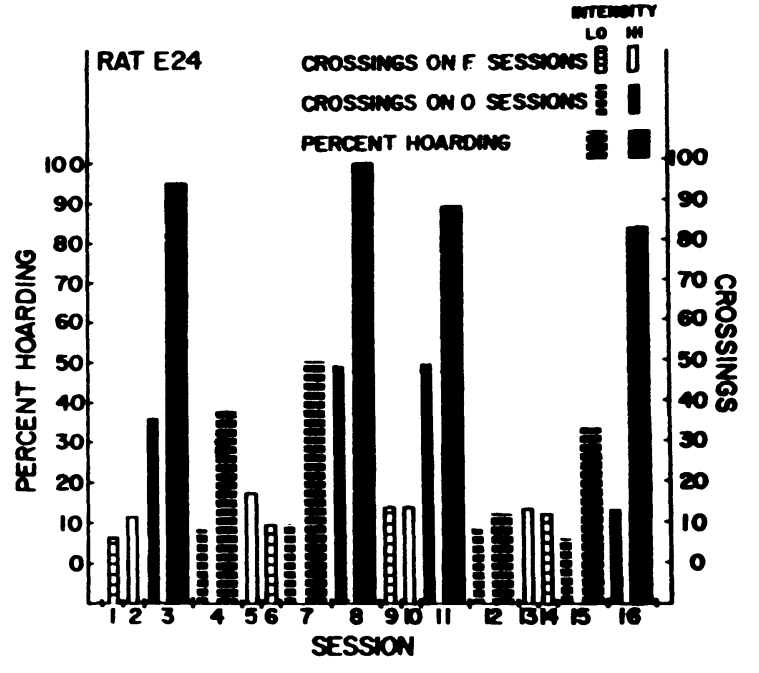

DISCUSSION

Some previous studies have left the impression that if a rat bears an electrode in the LHA and the ICS parameters are adjusted such as to induce the rat to turn ICS on and off by shuttling, then the rat will develop a tendency to carry objects from the OFF side to the ON side of the shuttlebox (Phillips et al, 1969; Valenstein et al, 1970). However, the results of the present experiment indicate that the fact that a rat engages in LHA shuttlebox self-stimulation is no guarantee that it will also carry objects. The neural states which support shuttling self-stimulation are not congruent with those that induce carrying behavior. The threshold intensities for these behaviors are different.

It is suggested here that, for carrying behavior to emerge, the current intensity must be high enough to potentially recruit a more intense or more immediate aversive neural process than that required for shuttlebox self-stimulation. The present results indicate that at lower intensities, even if they still support vigorous 
shuttling behavior, there may be a large decrement in carrying behavior. These results are consistent with the view that higher intensities of LHA ICS give rise to stronger or more immediate aversive effects and that these effects induce a stronger tendency to hoard in accordance with the security hypothesis.

\section{REFERENCES}

Beer. B.. Steiner. S.. \& Shaffer. M. M. The aversive properties of hrpothalamic stimulation. Communications in Behavioral Biology. Part B 1. No. 5. 1968. Abstract No. 05681162.

Bindra, D. What makes rats hoard? Journal of Comparative \& Physiological Psychology. 1948, 41, 397-402.

Bindra. D. Motivation: A sy'stematic re-interpretation. New York: Ronald Press. 1959.

Mendelson. J. Augmentation and diminution of the same reinforcing ICS by different ethological stimuli. Paper presented at the October 1966 meeting of the Psychonomic Society. St. Louis. Missouri.
Mendelson. J. Ecological modification of reinforcing effects of lateral hypothalamic stimulation in the rat. Paper presented at the April 1968 meeting of the Eastern Psychological Association. Washington. D.C.

Mendelson. J. Lateral hypothalamic stimulation: Inhibition of aversive effects by feeding. drinking. and gnawing. Scienci. 1969. 166. 1431-1433.

Mendelson. J. Ecological modulation of brain stimulation effects. International Journal of Psychobiology. 1972. 2. 285-304.

Phillips, A. G.. Cox, V. C.. Kakolewski. J. W.. \& Valenstein. E. S. Object-carrying by rats: An approach to the behavior produced by brain stimulation. Science. 1969. 166. 903-904.

Stein. L. An analysis of stimulus-duration preference in self-stimulation of the brain. Journal of Comparative \& Physiological Psychology. 1962, 55. 405-414.

Valenstein. E. S., Cox. V. C., \& Kakolewski. J. II. Re-examination of the role of the hypothalamus in motivation. Psychological Review, 1970. 77. 16-31.

(Received for publication October 16. 1972: revision accepted January 25. 1973.) 